\title{
Active versus passive adverse event reporting after pediatric chiropractic manual therapy: study protocol for a cluster randomized controlled trial
}

\author{
Katherine A. Pohlman ${ }^{1 *}$, Linda Carroll${ }^{2}$, Ross T. Tsuyuki ${ }^{3}$, Lisa Hartling ${ }^{4}$ and Sunita Vohra ${ }^{5}$
}

\begin{abstract}
Background: Patient safety performance can be assessed with several systems, including passive and active surveillance. Passive surveillance systems provide opportunity for health care personnel to confidentially and voluntarily report incidents, including adverse events, occurring in their work environment. Active surveillance systems systematically monitor patient encounters to seek detailed information about adverse events that occur in work environments; unlike passive surveillance, active surveillance allows for collection of both numerator (number of adverse events) and denominator (number of patients seen) data.

Chiropractic manual therapy is commonly used in both adults and children, yet few studies have been done to evaluate the safety of chiropractic manual therapy for children. In an attempt to evaluate this, this study will compare adverse event reporting in passive versus active surveillance systems after chiropractic manual therapy in the pediatric population.

Methods/design: This cluster randomized controlled trial aims to enroll 70 physicians of chiropractic (unit of randomization) to either passive or active surveillance system to report adverse events that occur after treatment for 60 consecutive pediatric (13 years of age and younger) patient visits (unit of analysis). A modified enrollment process with a two-phase consent procedure will be implemented to maintain provider blinding and minimize dropouts. The first phase of consent is for the provider to confirm their interest in a trial investigating the safety of chiropractic manual therapy. The second phase ensures that they understand the specific requirements for the group to which they were randomized. Percentages, incidence estimates, and 95\% confidence intervals will be used to describe the count of reported adverse events in each group. The primary outcome will be the number and quality of the adverse event reports in the active versus the passive surveillance group. With $80 \%$ power and $5 \%$ one-sided significance level, the sample size was calculated to be 35 providers in each group, which includes an 11\% lost to follow-up of chiropractors and 20\% of patient visits.
\end{abstract}

Discussion: This study will be the first direct comparison of adverse event reporting using passive versus active surveillance. It is also the largest prospective evaluation of adverse events reported after chiropractic manual therapy in children, identified as a major gap in the academic literature.

Trial registration: ClinicalTrials.gov, ID: NCT02268331. Registered on 10 October 2014.

Keywords: Pediatrics, Adverse event, Active surveillance, Passive surveillance

\footnotetext{
* Correspondence: kpohlman@parker.edu

'Research Institute, Parker University, 2540 Walnut Hill Lane, Dallas, TX 75229,

USA

Full list of author information is available at the end of the article
} 


\section{Background}

\section{Pediatric chiropractic manual therapy and patient safety}

Chiropractic manual therapy usually involves the therapeutic application of a force to a pre-determined body structure, which is typically a vertebral or extremity joint. There are numerous manual therapy variations with the velocity, amplitude, loading frequency, choice of lever, location, direction of load, and treatment frequency changing widely amongst the variations [1]. Spinal manipulation therapy (SMT), a type of manual therapy, is regulated for use in many professions (e.g., doctor of osteopathy, medical physicians, and physical therapists), but doctors of chiropractic (DCs) are the most likely to use SMT on a regular basis [2]. According to a 2015 practice analysis of United States DCs, 17.1\% of chiropractic patients are 17 years of age or less; this increases to $38.7 \%$ amongst DCs who specialize in pediatrics [2, 3].

Adverse events after manual therapy, including SMT, have been investigated more thoroughly in adult patients than in children [4-7]. Several reviews of adverse events in children following manual therapy have identified rare serious adverse events, although the studies have been primarily case reports. The main conclusion from these reviews was that there is insufficient primary research on this topic in this population [8-10].

\section{Patient safety performance - surveillance systems}

To measure safety performance, including reporting of adverse events, many health care settings have implemented surveillance systems to report and learn from adverse events. When established, such systems can provide learning opportunities based on the information gathered [11].

These patient safety surveillance systems vary according to their purpose. Active surveillance systematically collects information from the provider about patient encounters, including adverse events, which enhances reporting and demonstrates a health care organization's commitment to patient safety [11]. Although active surveillance can generate higher quality and quantity of reports because both numerator and denominator data are known, the time and resources needed to properly execute an active surveillance reporting system are often limitations to its successful implementation.

Passive surveillance voluntarily collects adverse event information from the provider and is more commonly utilized throughout health care [12]. Typically, passive surveillance systems are conducted confidentially and sometimes anonymously, and some have been modified for Internet-based fora. These systems can also promote quality improvement by allowing for reporting of adverse events, near misses (an event that could have caused an adverse event, but did not), and unsafe conditions. Passive surveillance systems are relatively easy to implement and can collect reports from a broad range of topics and individuals [12]. However, their major limitations include under-reporting (quantity of reports), inadequate information (quality of reports), and limited knowledge of how many patients were exposed (denominator data). Practitioners involved with passive surveillance systems have reported that they commonly forget to write-up their report, are too busy to review others' reports, are not sure who is responsible to writeup a report, or do not report an event because it seemed trivial [13].

\section{Study justification}

Within the chiropractic profession, active surveillance reporting systems are not used routinely. A passive surveillance system for chiropractic care, called the "Chiropractic Patient Incident Reporting and Learning System" (CPiRLS), is currently being used in Europe and Australia [14, 15]. Although CPiRLS does not have any age restrictions, to date only limited pediatric data have been reported into the system, despite multiple calls for high-quality safety data about pediatric chiropractic manual therapy $[8,10]$.

Both active and passive surveillance methods have distinct advantages and limitations. The need for a direct comparison of the ability of active versus passive surveillance to report adverse events, and the need to better understand the patient safety performance in the use of chiropractic manual therapies for the pediatric population, led to the development of this cluster randomized clinical trial.

\section{Study aim and hypothesis}

Study aim: to compare the quantity and qualify adverse event reports after chiropractic manual therapy in children 13 years of age or under, using passive versus active surveillance reporting systems. Hypothesis: DCs randomized to the active surveillance system will report more adverse events and will have better quality reporting than those randomized to the passive surveillance system.

\section{Methods}

\section{Study design}

The study design is a pragmatic, superiority, cluster randomized clinical trial with a modified enrollment process to maintain participant blinding. DCs in private practice who treat children will be the unit of randomization with random allocation in a 1:1 ratio to active or passive surveillance reporting systems. Cluster randomization was chosen for practical reasons with the unit of analysis being reports from the individual chiropractic patient visits. The University of Alberta's Research Ethics Board reviewed and approved this study (Pro00027903). The trial has been registered at ClinicalTrials.gov (NCT02268331). The study 
protocol was prepared using the Standard Protocol Items: Recommendations for Interventional Trials (SPIRIT) guidelines [16] (see Additional file 1) and also the "Methods" section of the Consolidated Standards of Reporting Trials (CONSORT) 2010 Checklist for reporting a cluster randomized controlled trial [17] (see Additional file 2).

\section{Recruitment, randomization, and enrollment}

Licensed DCs in the United States and Canada will be recruited from a variety of venues, including pediatric chiropractic-specific events and organizations, social media, and professional newsletters/magazines. Word of mouth and referrals from colleagues and past participants will also be source of referral into the study.

As shown in Fig. 1, DCs interested in the study will complete a demographic questionnaire and review/sign the initial consent document, which states that they are interested in enrolling in a study to report safety information from 60 consecutive pediatric visits. They will then be randomized to passive or active surveillance by the study coordinator (KAP). To promote baseline equivalence, we

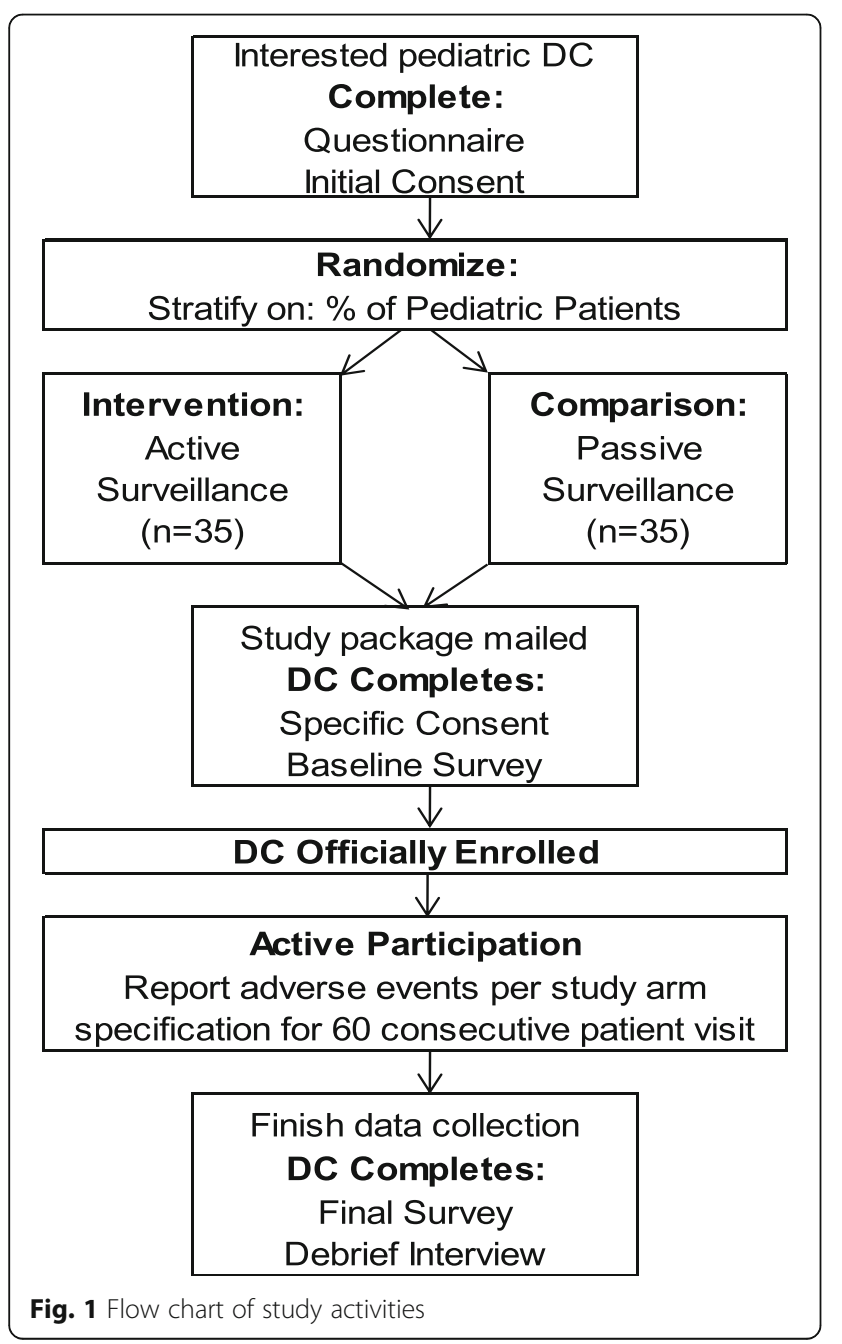

will stratify by DC's self-reported average proportion of pediatric patients seen $(>20 \%$ versus $\leq 20 \%)$. To maintain allocation concealment, the REDCap (Research Electronic Data Capture) Randomization Module will be utilized with a random, variable, permuted block size, generated by an independent biostatistician [18]. Interested DCs will have study materials sent directly to their offices. This material includes the Consent Form that gives details on the surveillance system to which they were randomly assigned. DCs are considered enrolled in the study after that Consent Form is signed and they complete the online baseline survey, which collects additional demographic data and assesses patient safety attitudes [19]. Throughout study participation, to ensure compliance with study methods, regular communications will occur via email or telephone between the study coordinator (KAP) and the DC.

\section{Intervention arm: active surveillance}

For 60 consecutive child patient visits, the parents/caregivers will be given an Information Sheet and asked to complete a pre-treatment form before the child sees the DC. As described in the Information Sheet and as stated on the top of all data collection forms, consent will be implied if the data collection forms are completed and returned. This ensures patient confidentiality. Patients and providers will each be given a post-treatment form to complete. The patient's post-treatment form is to be completed within 1 week and returned directly to the investigators using a postage-paid envelope. The DC's post-treatment form is to be completed immediately after the patient's visit. A more detailed form documenting adverse events will be completed by the provider if a moderate, serious or severe adverse event (see definitions in Table 1) occurs immediately following treatment or is reported to the DC at a later date. All forms (see Additional file 3) were modified from an ongoing, active surveillance study on SMT in Canada [20]. The modified forms were reviewed for content validation by a group of experts, which included the original developers, pediatric chiropractic experts, and caregivers of pediatric chiropractic patients.

\section{Comparison arm: passive surveillance}

The passive surveillance system will use the established Chiropractic Patient Incident Reporting and Learning System (CPiRLS) [15]. DCs will be asked to report adverse events that occur in 60 consecutive pediatric patient visits. In this system, only registered providers can submit, read or comment on reports. Participating DCs will be given a universal code to protect anonymity and will also be provided with the CPiRLS's "trigger list" to advise on what kinds of incidents/adverse events should be reported (see Additional file 4). Reports and comments submitted will be 
Table 1 Definitions of terminology for study protocol [20]

\begin{tabular}{|c|c|}
\hline $\begin{array}{l}\text { Adverse event } \\
\text { (AE) }\end{array}$ & $\begin{array}{l}\text { Any unfavorable sign, symptom or disease } \\
\text { temporally associated with the treatment, } \\
\text { whether or not caused by the treatment. } \\
\text { Specifically, any new symptom of moderate severity } \\
\text { or a pre-existing symptom that is worse after treatment }\end{array}$ \\
\hline \multirow[t]{4}{*}{ Seriousness } & $\begin{array}{l}\text { Mild: asymptomatic or mild symptoms, self- } \\
\text { care only (e.g., ice/heat, over-the-counter } \\
\text { analgesic) }\end{array}$ \\
\hline & $\begin{array}{l}\text { Moderate: limiting age-appropriate activities } \\
\text { of daily living (e.g., work, school); or sought } \\
\text { care from a physician }\end{array}$ \\
\hline & $\begin{array}{l}\text { Severe: medically significant but not immediately } \\
\text { life-threatening; temporarily limits self-care (e.g., } \\
\text { bathing, dressing, eating) (for } 5 \text { years of age and } \\
\text { older); or urgent or emergency room assessment } \\
\text { sought }\end{array}$ \\
\hline & $\begin{array}{l}\text { Serious: results in death or a life-threatening adverse } \\
\text { event or an adverse event resulting in inpatient } \\
\text { hospitalization or prolongation of existing } \\
\text { hospitalization for more than } 24 \text { h: a persistent } \\
\text { or significant incapacity or substantial disruption } \\
\text { of the ability to conduct normal life functions; a } \\
\text { congenital anomaly/birth defect }\end{array}$ \\
\hline \multirow[t]{4}{*}{$\begin{array}{l}\text { Causality (i.e., } \\
\text { relatedness) }\end{array}$} & $\begin{array}{l}\text { Certain: a clinical event occurring in a } \\
\text { plausible time relationship to treatment, } \\
\text { and which cannot be explained by } \\
\text { concurrent disease or other drugs or } \\
\text { therapies }\end{array}$ \\
\hline & $\begin{array}{l}\text { Probable/likely: a clinical event with a reasonable } \\
\text { time sequence to treatment, unlikely to be } \\
\text { attributed to concurrent disease or other } \\
\text { drugs or therapies }\end{array}$ \\
\hline & $\begin{array}{l}\text { Possible: a clinical event with a reasonable } \\
\text { time sequence to treatment, but which } \\
\text { could also be explained by concurrent } \\
\text { disease or other drugs or therapies }\end{array}$ \\
\hline & $\begin{array}{l}\text { Unlikely: a clinical event with a temporal } \\
\text { relationship to treatment which makes a } \\
\text { causal relationship improbable, and in } \\
\text { which drugs, other therapies or underlying } \\
\text { disease provide plausible explanations }\end{array}$ \\
\hline \multirow[t]{6}{*}{ Preventability } & 1: Virtually no evidence of preventability \\
\hline & 2: Slight to modest evidence of preventability \\
\hline & $\begin{array}{l}\text { 3: Preventability not quite likely (less than } \\
50 / 50 \text {, but "close call") }\end{array}$ \\
\hline & $\begin{array}{l}\text { 4: Preventability more than likely (more than } \\
50 / 50 \text {, but "close call") }\end{array}$ \\
\hline & 5: Strong evidence of preventability \\
\hline & 6: Virtually certain evidence of preventability \\
\hline \multirow[t]{7}{*}{ Patient disposition } & 1: Resolved, no sequelae \\
\hline & 2: AE still present - no treatment \\
\hline & 3: AE still present - being treated \\
\hline & 4: Residual effects present - no treatment \\
\hline & 5: Residual effects present - treated \\
\hline & 6: Death \\
\hline & 7: Unknown \\
\hline
\end{tabular}

monitored by both the CPiRLS team and the study's investigators.

\section{Adjudication}

In both the active and the passive groups, when a moderate, severe or serious adverse event is identified, all information from the report will be reviewed independently by blinded content experts to evaluate the event according to the terminology outlined in Table 1 (causality, preventability, and patient disposition). Operational definitions for all terminology were determined through a consensus-based process by the SafetyNET team of manual therapy and patient safety experts $[20,21]$.

\section{Outcomes}

The primary outcome will be the number (the count) and quality (i.e., ability to meaningfully interpret/adjudicate, a binary variable) of the DC's adverse event(s) reports per patient visit and per patient in each group. Quality of adverse event reports will be assessed by the adjudicators' ability to meaningfully adjudicate the report (section above).

A secondary outcome is the change in patient safety attitudes for participating DCs. This will be measured in both groups using the Safety Organizing Scale [19], which is a nine-item survey with a 7-point rating scale $(1-$ "Not at all"; 7 - "To a very great extent"). This questionnaire is to be completed at two time points: at baseline (the online baseline survey prior to study enrollment) and after adverse event data collection is complete for each participating DC. In the active surveillance arm, additional variables to assess adverse events and associated factors for adverse events include: patient-reported adverse events, manual therapy treatment description, patient health history, and patient satisfaction [22].

\section{Minimization of systematic error}

To reduce potential respondent bias and maximize data integrity, a modified enrollment process will be utilized with a two-phased consent process. The first phase has a consent document focused on safety outcomes data collection rather than a comparison of the two different methodologies for collecting such outcomes. This focus is utilized to both blind participants to the comparison under evaluation and minimize dropouts as one arm (active surveillance) is more time intensive than the other (passive surveillance), but both arms are enhancements to current standard of North America practices. The second phase occurs after randomization with the consent document explaining the exact study procedures of the participant's allocated group without reference to the other group. There will be a debrief interview at the end of a DC's study participation to explain this 
modified enrollment process and the procedure for both study groups.

Other study personnel who will be blinded in the study include: (1) patients, (2) an independent biostatistician for analysis, and (3) content experts involved in the adjudication process. Because of the major differences in data management, the investigator (KAP) responsible for study coordination cannot be blinded.

\section{Clinical data management}

All data will be entered and managed using REDCap electronic data capture tools, which is hosted at the University of Alberta [18]. REDCap is a secure, web-based application designed to support data capture for research studies.

For the active surveillance group, the data will be verified and validated, and the quality checked by a single study investigator (KAP) who will compare the patient's pre- and post-treatment forms to ensure that inconsistencies are corrected. For audit purposes and to ensure transparency, all changes made will be recorded with the time and date and user ID. The study investigator will discuss any queries with the study team with query resolutions recorded.

\section{Statistical methods}

The count of reported adverse events (any severity) in each group will be expressed with percentages and incidence estimates, and their 95\% confidence intervals (CIs). The primary analysis will compare the cumulative incidence of adverse event reports in active versus passive surveillance. Because the outcome is number of events, it is assumed that the data will follow a Poisson distribution. Hence, a Poisson regression with log links will be used in general estimating equation (GEE) analyses with an appropriate sandwich estimator to take into account the DC cluster correlation. Groups will be compared using an intention-to-treat analysis.

Sensitivity analysis, using the same GEE analyses as above, will be conducted for reports that were not adjudicated (because of uninterpretable adverse events) and differences in how missing data were handled (i.e., imputing using average incidence and highest incidence). The binary variable expressing if the quality of the adverse event report allowed for meaningful interpretation/adjudication will be evaluated using the McNemar's exact test because of the expected rarity of reports and cluster correlation.

Secondary analysis will address differences in the count of adverse event reports by patient-only, provideronly, and those reported by the active surveillance versus the provider-reports in the passive surveillance. Like the primary analyses, Poisson regression with log links will be used in GEE analyses to account for cluster-specific methods. Patient safety attitudes will be measured before and after participation and compared across surveillance groups.

Other planned secondary analyses are designed to identify factors associated with adverse events from the data gathered in the active surveillance group. Potential factors for adverse events include patient characteristics (e.g., age, presenting condition, sex, health history), provider characteristics (e.g., years in practice, specialty training), and treatment provided (e.g., high-velocity, low-amplitude or other). With the adverse event reports categorized by their severity (i.e., none, mild, moderate, severe, serious), logistic regression analyses will be used to model factors associated with the adverse events. If the number of moderate, severe, and serious events are small, the outcome will be dichotomized as any adverse event versus no adverse event. If numbers of moderate, severe and serious events are sufficiently large, multivariable polytomous logistic regression will be used.

Planned exploratory analyses include: (1) subgroup analysis for providers with a specialty pediatric certification and number of reported adverse events (i.e., the primary outcome); (2) assessment of the feasibility to implement a surveillance system within chiropractic offices from individual provider feedback; and (3) review of debrief interview to gain insight into participating DCs' overall thoughts on the study, including barriers to implementation, perceived benefit of participating, and being blinded to intervention. An assessment of bias will be conducted with responding and non-responding patient demographic characteristics for the active surveillance group. All analyses will be conducted using Stata version 13 (StataCorp LP, College Station, TX, USA).

\section{Sample size}

An estimated active surveillance reporting rate of $4.3 \%$ and intracluster correlation of $\rho=0.13$ were based on a pilot study of a similar active surveillance used within the chiropractic profession in Canada [20, 21]. We assumed a passive surveillance reporting rate of $0.53 \%$, based on prior academic literature [8]. A one-sided significance level was utilized as it seems reasonable to believe that passive surveillance will result in underreporting of adverse events [23]. We calculated that a sample size of 35 providers in each group, with each DC collecting data from 60 pediatric patient visits, and 5\% one-sided significance level, would lead to $80 \%$ power. This includes an anticipated loss to follow-up of $11 \%$ of DCs and $20 \%$ of patient visits.

\section{Discussion}

This study will be the largest prospective evaluation of adverse events reported after chiropractic manual therapy in the pediatric population, which has been identified as a major gap in the academic literature [8-10, 24]. This randomized cluster trial assesses the effectiveness 
of two different surveillance methods to collect observational safety data on a topic that is clinically relevant. To our knowledge, this is the first study to do a direct comparison of active versus passive surveillance reporting of adverse events.

The chiropractic profession treats children [3, 25]; therefore, it has a responsibility to ensure proper safety evaluations. The attitudes and opinions of DCs, who are interested in pediatric treatment, for implementing safety performance systems were evaluated in 2014. The survey identified a robust patient safety climate with time pressure as the barrier of most concern to participants [26]. Time pressure is a common barrier for health care provider participation in research, as "busy-ness" is seen as a socially acceptable excuse for declining "extra" activities [27]. Our study protocol took this concern into consideration. When pilot tested, passive surveillance was found to add $30 \mathrm{~s}$ per patient visit while active surveillance added only 2 min [20].

Aside from reports of actual adverse events that are collected in this study, each surveillance method also collects additional patient safety information. While not the primary outcome, this study will also clearly describe and report these differences. Such examples from the passive surveillance group includes administrative, incidental patient safety incidents (e.g., use of the wrong clinical file or tripping over office equipment) or "near misses"/events, which could have caused an adverse event, but did not. For the active surveillance group, information will be sought not only from the DC, but also directly from the patients; patient-provided information can be compared to that information known by the provider. These differences are unique to each surveillance group and should be taken in consideration when an organization is deciding on what method to use to evaluate adverse event.

Beyond the significance of the study's specific aims, the study procedures also include several notable methodological considerations, such as the attention to outcome measurement and a modified enrollment process to maintain participant blinding. This study started with a content validation of the data collection instruments to ensure that they will collect the intended information and that it will be easily understood by the chiropractic pediatric patient's parent/caregiver [28].

Modified enrollment procedures have been utilized most commonly to avoid biases that occur with nonplacebo-controlled trials [29]. This study will use a modified enrollment procedure, a two-stage consent process, to ensure that provider blinding is maintained and dropouts minimized. To avoid ethical concerns regarding enrolling and randomizing providers without their consent, consent is sought in two stages: first, providers consent to participation in a study on pediatric patient safety and chiropractic manual therapy. The second consent will give full disclosure of their specific study procedures. When participant's complete the study, a debrief interview will unveil the two groups and the purpose for not disclosing this information earlier.

\section{Barriers to study completion}

Possible barriers to the study's implementation will be the willingness of DCs to participate in research and their adherence to study procedures. Adherence will be addressed by actively following up on DCs interested in this study's topic, engaging front desk personnel in study processes, and assuring that the study protocol is understood. Despite these precautions, compliance is expected to be challenging, specifically for chiropractic practices that are assigned to the active surveillance group. Dropouts have been taken into account in the sample size calculations.

Another concern regarding the study's implementation is the possibility of a low response rate for the active surveillance arm's post-treatment form, to be completed by the patient's caregiver. The pilot study found that DCs who encouraged their patients to complete the data collection instruments had a better response rate [20,21].

\section{Trial status}

This is the first version of the study protocol. Modification or amendments that have an impact on the conduct of the study will be documented and described in further publications. At the time of protocol submission, this trial was in active recruitment.

\section{Additional files}

\author{
Additional file 1: SPIRIT 2013 Checklist. (PDF $48 \mathrm{~kb}$ ) \\ Additional file 2: CONSORT 2010 Checklist. (PDF 137 kb) \\ Additional file 3: Active Surveillance Study Forms. (PDF 23072 kb) \\ Additional file 4: Passive Surveillance Trigger List. (PDF 365 kb)
}

\section{Abbreviations}

CPiRLS: Chiropractic Patient Incident Reporting and Learning System; DC: Doctor of chiropractic; GEE: General estimating equation;

REDCap: Research Electronic Data Capture; SMT: Spinal manipulation therapy

\section{Acknowledgements}

This project was done as part of a team grant, SafetyNET; the authors would like to thank all principal investigators, Drs. Kawchuk, Caulfield, Boon, and O'Beirne, as well as members of Team \#3 (M Funabashi, for their insightful comments and suggestions to improve the project). In addition, the authors would like to acknowledge the ACA and ICA Council on Chiropractic Pediatrics for their support reviewing the protocol and with recruitment of physician. Lastly, the authors acknowledge, Dr. Elise Hewitt, a pediatric chiropractic specialist who supported the study design upon inception, was instrumental in pilot testing and recruitment, and is a leader and mentor within the chiropractic profession.

\section{Funding}

This study has been supported by the Women's and Children's Health Research Institute, University of Alberta (RES0013182) and the Canadian Institutes of Health Research (TGSTAIR 112758). L Carroll and S Vohra 
received salary support as an Alberta Innovates-Health Solutions: Health Senior Scholar and Health Scholar, respectively.

\section{Availability of data and materials}

Not applicable

\section{Authors' contributions}

KAP, SV, and LC were responsible for study conception. Trial design was the responsibility of all authors. KAP was responsible for conducting the trial and writing the manuscript with critical review and contributions by SV, LC, RT, and LH. All authors read and approved the final manuscript.

\section{Ethics approval and consent to participate}

The University of Alberta's Research Ethics Board reviewed and approved this study (Pro00027903); no other ethical review is necessary as each participant was in private practice. Informed consent will be obtained from all participants.

\section{Consent for publication}

Not applicable

\section{Competing interests}

The authors declare that they have no competing interests.

\section{Publisher's Note}

Springer Nature remains neutral with regard to jurisdictional claims in published maps and institutional affiliations.

\section{Author details}

${ }^{1}$ Research Institute, Parker University, 2540 Walnut Hill Lane, Dallas, TX 75229, USA. ${ }^{2}$ School of Public Health, University of Alberta, 3-300 Edmonton Clinic Health Academy, 11405-87 Ave, Edmonton, AB T6G 1C9, Canada. ${ }^{3}$ EPICORE CENTRE, Faculty of Medicine and Dentistry, University of Alberta, 362 Heritage Medical Research Centre, Edmonton, AB T6G 2S2, Canada. ${ }^{4}$ Department of Pediatrics, Faculty of Medicine and Dentistry, University of Alberta, 4-472 Edmonton Clinic Health Academy, 11405-87 Ave, Edmonton, AB T6G 1C9, Canada. ${ }^{5}$ CARE Program, Department of Pediatrics, Faculty of Medicine and Dentistry, University of Alberta, Suite \#1702, College Plaza, 8215112 St. NW, Edmonton, AB T6G 2C8, Canada.

Received: 24 October 2016 Accepted: 21 October 2017

Published online: 01 December 2017

\section{References}

1. Triano JJ. Clinical biomechanics of spinal manipulation. In: Herzog W, editor. The mechanics of spinal manipulation. New York: Churchill Livingstone; 2000. p. 92-190.

2. Christensen M, Kollasch M, Hyland J. Practice analysis of chiropractic 2010: a project report, survey analysis, and summary of chiropractic practice in the United States. Greenley, CO: National Board of Chiropractic Examiners; 2010.

3. Pohlman KA, Hondras MA, Long CR, Haan AG. Practice patterns of doctors of chiropractic with a pediatric diplomate: a cross-sectional survey. BMC Complement Altern Med. 2010;10:26-6882-10-26

4. Rubinstein SM. Adverse events following chiropractic care for subjects with neck or low-back pain: do the benefits outweigh the risks? J Manipulative Physiol Ther. 2008:31(6):461-4.

5. Cagnie B, Vinck E, Beernaert A, Cambier D. How common are side effects of spinal manipulation and can these side effects be predicted? Man Ther. 2004;9(3):151-6.

6. Assendelft WJJ, Bouter LM, Knipschild PG. Complications of spinal manipulation. J Fam Pract. 1996:42(5):475-80.

7. Cassidy JD, Boyle E, Cote P, He Y, Hogg-Johnson S, Silver FL, Bondy SJ. Risk of vertebrobasilar stroke and chiropractic care: results of a population-based case-control and case-crossover study. Spine. 2008:33(4 Suppl):S176-83.

8. Todd AJ, Carroll MT, Robinson A, Mitchell EK. Adverse events due to chiropractic and other manual therapies for infants and children: a review of the literature. J Manipulative Physiol Ther. 2015;38(9):699-712.

9. Humphreys BK. Possible adverse events in children treated by manual therapy: a review. Chiropr Osteopat. 2010;18:12.
10. Vohra S, Johnston BC, Cramer K, Humphreys K. Adverse events associated with pediatric spinal manipulation: a systematic review. Pediatrics. 2007;119(1):e275-83.

11. Kohn LT, Corrigan J, Donaldson MS. To err is human: building a safer health system. Washington, D.C.: National Academy Press; 2000.

12. Ferranti J, Horvath MM, Cozart H, Whitehurst J, Eckstrand J. Reevaluating the safety profile of pediatrics: a comparison of computerized adverse drug event surveillance and voluntary reporting in the pediatric environment. Pediatrics. 2008;121(5):e1201-7.

13. Evans SM, Berry JG, Smith BJ, Esterman A, Selim P, O'Shaughnessy J, DeWit M. Attitudes and barriers to incident reporting: a collaborative hospital study. Qual Saf Health Care. 2006;15(1):39-43.

14. Thiel $\mathrm{H}$. Incident reporting and learning systems for chiropractors developments in Europe. J Can Chiropr Assoc. 2011;55(3):155-8.

15. Thiel $\mathrm{H}$, Bolton J. The reporting of patient safety incidents - first experiences with the chiropractic reporting and learning system (CRLS): a pilot study. Clin Chiropr. 2006;9:139-49.

16. Chan AW, Tetzlaff JM, Altman DG, Laupacis A, Gotzsche PC, Krle A-Jeric K, Hrobjartsson A, Mann H, Dickersin K, Berlin JA, Dore CJ, Parulekar WR, Summerskill WS, Groves T, Schulz KF, Sox HC, Rockhold FW, Rennie D, Moher D. SPIRIT 2013 Statement: defining standard protocol items for clinical trials. Ann Intern Med. 2013:158:200-7.

17. Campbell MK, Piaggio G, Elbourne DR, Altman DG, CONSORT Group. Consort 2010 statement: extension to cluster randomised trials. BMJ. 2012;345:e5661

18. Harris PA, Taylor R, Thielke R, Payne J, Gonzalez N, Conde JG. Research electronic data capture (REDCap) — a metadata-driven methodology and workflow process for providing translational research informatics support. J Biomed Inform. 2009;42(2):377-81.

19. Vogus TJ, Sutcliffe KM. The Safety Organizing Scale: development and validation of a behavioral measure of safety culture in hospital nursing units. Med Care. 2007:45(1):46-54.

20. Pohlman KA, O'Beirne M, Thiel H, Cassidy JD, Mior S, Hurwitz EL, Westaway M, Ishaque S, Yager JY, Vohra S. Development and validation of providers' and patients' measurement instruments to evaluate adverse events after spinal manipulation therapy. Eur J Integr Med. 2014;6(4):451-66.

21. Vohra S, Kawchuk G, Caulfield T, Pohlman K. SafetyNET: an interdisciplinary team supporting a safety culture for spinal manipulation therapy. Eur J Integr Med. 2014:6(4):473-7.

22. Cherkin DC, Sherman KJ, Kahn J, Erro JH, Deyo RA, Haneuse SJ, Cook AJ. Effectiveness of focused structural massage and relaxation massage for chronic low back pain: protocol for a randomized controlled trial. Trials. 2009;10:96.

23. Stockwell DC, Kane-Gill SL. Developing a patient safety surveillance system to identify adverse events in the intensive care unit. Crit Care Med. 2010; 38(6 Suppl):S117-25

24. Humphreys BK. Possible adverse events in children treated by manual therapy: a review. Chiropr Osteopat. 2010;18:7.

25. Christensen M, Hyland J, Goertz C, Kollasch M. Practice analysis of chiropractic 2015: a project report, survey analysis, and summary of chiropractic practice in the United States. Greeley, CO: National Board of Chiropractic Examiners; 2015

26. Pohlman KA, Carroll L, Hartling L, Tsuyuki RT, Vohra S. Barriers to implementing a reporting and learning patient safety system: pediatric chiropractic perspective. J Evid Based Complementary Altern Med. 2016;21(2):105-9.

27. Cvijovic K, Boon H, Jaeger W, Vohra S. Pharmacists' participation in research: a case of trying to find the time. Int J Pharm Pract. 2010;18(6):377-83.

28. Mokkink LB, Terwee CB, Knol DL, Stratford PW, Alonso J, Patrick DL, Bouter LM, de Vet HC. The COSMIN checklist for evaluating the methodological quality of studies on measurement properties: a clarification of its content BMC Med Res Methodol. 2010;10:22-2288-10-22.

29. Adamson J, Cockayne S, Puffer S, Torgerson DJ. Review of randomised trials using the post-randomised consent (Zelen's) design. Contemp Clin Trials. 2006:27(4):305-19. 